\title{
Une approche technique et rationaliste
}

La formation des enseignants en Angleterre

A technical-rationalist approach. Teacher Training in England

Un enfoque técnico y racionalista. La formación de los profesores en Inglaterra

\section{Olwen McNamara}

Traducteur : Jérôme Quintana

\section{OpenEdition}

\section{Journals}

Édition électronique

URL : http://journals.openedition.org/ries/933

DOI : $10.4000 /$ ries.933

ISSN : 2261-4265

Éditeur

Centre international d'études pédagogiques

Édition imprimée

Date de publication : 1 décembre 2010

Pagination : 49-60

ISBN : 978-2-8542-583-1

ISSN : 1254-4590

Référence électronique

Olwen McNamara, « Une approche technique et rationaliste », Revue internationale d'éducation de Sèvres [En ligne], 55 | décembre 2010, mis en ligne le 01 décembre 2013, consulté le 02 mai 2019 URL : http://journals.openedition.org/ries/933 ; DOI : 10.4000/ries.933 


\title{
Une approche technique et rationaliste
}

\section{La formation des enseignants en Angleterre*}

\author{
Olwen McNamara
}

\section{HISTORIQUE}

La formation des enseignants en Angleterre a débuté dans les collèges universitaires confessionnels à la fin du XIX ${ }^{\mathrm{e}}$ siècle, puis très vite dans les départements d'éducation des universités, qui traditionnellement ne proposaient une formation, après l'obtention du diplôme universitaire, qu'aux enseignants des établissements privés et sélectifs. En 1969, une formation obligatoire a été mise en place, destinée aux diplômés désireux d'enseigner dans le primaire, puis dans le secondaire à partir des années 1970. Suite à la mise en place généralisée de cette formation au métier d'enseignant, on a assisté dans les années soixante-dix et quatre-vingt à une longue série de fusions institutionnelles qui ont transformé les écoles normales en instituts polytechniques (devenus universités en 1992) et en universités. La perte d'autonomie qui en a résulté concernait les écoles normales, qui s'attendaient toutefois en contrepartie à être davantage protégées contre les excès du gouvernement en matière de régulation. Ces attentes se sont avérées infondées.

Le dernier quart de siècle a vu naître de profonds changements, à la fois en raison de l'importance du micro-management dans le secteur de l'éducation mais aussi en raison de l'ampleur, du rythme et du caractère radical du programme de réformes. Au Royaume-Uni, cette réforme a touché principalement l'Angleterre. Dans les quatre nations britanniques, la tendance est à une divergence croissante des politiques menées en matière de formation des enseignants, et d'éducation en général, depuis la dévolution du pouvoir exécutif au Pays de Galles et du pouvoir législatif au Parlement écossais et à l'Assemblée d'Irlande du Nord en 1992. Néanmoins, l'Écosse tout particulièrement s'est toujours distinguée sur le plan de l'éducation. Les quatre nations possèdent aujourd'hui une réglementation différente et, comme le notait Robin Alexander en 1984, les deux influences déterminantes dans le domaine de la formation initiale résident dans la culture et l'organisation des instituts de formation des enseignants et dans leur relation avec les instances de validation. Ces deux influences, et notamment la deuxième, ont joué un rôle crucial dans les développements qui se sont opérés en Angleterre.

* Article traduit de l'anglais par Jérôme Quintana. 


\section{Politisation}

Le mouvement en faveur d'une centralisation et d'un contrôle politique de la formation des enseignants s'est manifesté pour la première fois en 1984 lors de la création du Conseil pour l'accréditation de la formation des enseignants (Council for Accreditation of Teacher Education). Le Conseil s'est efforcé d'apporter plus de rigueur dans la façon dont les formations étaient évaluées et dispensées. Mais il a surtout également cherché à mettre en place une formation plus pratique en établissement scolaire, qui comprenne des parcours axés sur l'école. Pour ce qui est de ces parcours, dans la décennie qui a suivi, et ce en dépit des efforts déployés, le pourcentage général de stagiaires suivant des parcours de formation axés sur l'école n'avait toujours pas augmenté de façon remarquable (Furlong, Barton, Whiting, 2000). Toutefois, au milieu des années 1990, le Conseil a été remplacé par l'Agence de formation des enseignants (Teacher Training Agency), organisme (quasi) gouvernemental ne relevant d'aucun ministère et doté de pouvoirs beaucoup plus larges et importants. Son domaine de compétence se limitait à l'Angleterre et comprenait le financement, l'assurance et le contrôle qualité, l'accréditation des parcours de formation, le recrutement des enseignants et leur accueil. Le passage du Conseil à l'Agence a également marqué un changement dans l'idéologie et la gouvernance de la formation, et notamment une modification profondément symbolique de la désignation des termes en passant de «l'éducation » à la "formation » initiale des enseignants.

Quelques années plus tard, lorsque le nouveau gouvernement travailliste est arrivé au pouvoir, il a créé le Conseil général de l'enseignement pour l'Angleterre (General Teaching Council for England) comme instance de régulation indépendante, afin de relever le niveau de la profession, de tenir un registre des enseignants et d'établir un code de conduite professionnelle. L'Écosse avait depuis 1965 créé avec succès un Conseil du même ordre, mais son équivalent anglais n'a en revanche jamais suscité l'attrait des enseignants qui, déjà soumis à de nombreuses réglementations et inspections, n'attribuaient à ce Conseil qu'une fonction essentiellement disciplinaire et, pour couronner le tout, devaient verser une somme pour obtenir leur inscription au registre des enseignants. En 2010, durant ses premières semaines au gouvernement, la coalition conservatrice a annoncé de manière péremptoire l'abolition du Conseil général de l'enseignement. Il reste toutefois à savoir comment les fonctions juridiques de cet organisme seront désormais assurées.

Alors que le Conseil général de l'enseignement pour l'Angleterre continuait de lutter pour établir son autorité et avoir voix au chapitre, l'Agence pour la formation des enseignants est devenue le principal moteur du changement en Angleterre. Au départ, l'Agence était motivée par le besoin d'accroître le nombre d'enseignants, car peu de temps après sa création, elle a été confrontée à la plus grave pénurie d'enseignants de la période récente (1999-2002). Cela s'est traduit 
par une hausse rapide du nombre de stagiaires en formation (30\% dans le primaire, $50 \%$ dans le secondaire) pour atteindre le nombre record de 42000 stagiaires en 2003-2004. Cette pénurie a été provoquée par la hausse des effectifs scolaires, le faible taux de maintien en poste des professeurs et la baisse des recrutements. Elle s'est trouvée exacerbée lorsque le gouvernement a introduit en 1998 des frais de scolarité pour les étudiants préparant un diplôme de l'enseignement supérieur. Elle a également forcé le gouvernement à proposer des bourses de formation ainsi que d'autres mesures incitatives en 2002 aux stagiaires diplômés intégrant la formation initiale. Les mesures incitatives dans le cadre du recrutement étaient différenciées et ciblées selon les besoins. Par exemple, pour les matières déficitaires (comme les mathématiques ou les sciences au niveau secondaire), les bourses accordées aux stagiaires diplômés intégrant la formation en un an s'élèvent aujourd'hui à $9000 \mathfrak{E}^{1}$. Les stagiaires ont également droit à des primes d'installation lorsqu'ils prennent leurs fonctions d'enseignant. Les bourses octroyées aux stagiaires des matières non déficitaires au niveau secondaire, et au niveau primaire lorsque la concurrence est rude pour obtenir une place en formation, sont beaucoup plus faibles et ont été diminuées petit à petit jusqu'à atteindre le montant actuel de $4000 \mathfrak{E}$ par an ${ }^{2}$. Aujourd'hui, l'Angleterre arrive à la fin d'une période de sureffectif d'enseignants en raison d'une diminution des effectifs scolaires qui a entraîné d'importantes baisses des dotations, notamment pour les matières non déficitaires du secondaire. Cette diminution temporaire des effectifs scolaires doit cependant être mise en regard avec une croissance estimée de la population, selon un rapport récent du Bureau des statistiques nationales (Office of National Statistics), et avec l'imminent départ à la retraite d'un tiers des enseignants qui ont plus de 50 ans, sachant que $20 \%$ d'entre eux ont plus de 55 ans (GTCE, 2009). Néanmoins, il reste à voir quelle sera l'incidence des toutes récentes hausses de l'âge auquel on peut prétendre à la retraite, ainsi que de l'abolition de l'âge légal de départ à la retraite.

Outre la gestion des fluctuations dans les effectifs enseignants, l'Agence a également poursuivi un but idéologique. Elle s'est donné pour objectif tout d'abord de renforcer la diversité du vivier de candidats, afin que le corps enseignant soit plus représentatif de la population scolaire. Les enseignants noirs et ceux issus des minorités ethniques constituent aujourd'hui moins de $6 \%$ de la totalité du corps enseignant, alors que la proportion d'élèves noirs et issus des minorités ethniques dans le système éducatif a augmenté de façon rapide pour atteindre $17 \%$ dans le secondaire et $20 \%$ dans le primaire. Ensuite, elle s'est efforcée de promouvoir plus vigoureusement un catalogue de formation incluant des filières souples organisées en modules et permettant d'augmenter le nombre jusque là décevant de parcours "alternatifs » intégrés à l'enseignement, par le biais de programmes axés sur l'école et de parcours de formation 
en milieu professionnel. Même si la portée politique de l'introduction des parcours «alternatifs » dans l'enseignement en Angleterre au début des années 1990 a été considérable, dans la pratique ces parcours ont été très peu suivis, même à la fin de la décennie.

L'Agence a très nettement atteint le double objectif qu'elle s'était fixé. En premier lieu, la proportion de stagiaires noirs et issus des minorités ethniques a plus que doublé en une décennie. Elle atteint aujourd'hui $9 \%$ dans le primaire et $15 \%$ dans le secondaire. Deuxièmement, le nombre total de stagiaires suivant des parcours de formation en milieu professionnel a été multiplié par six, le principal étant le Graduate Teacher Programme, d'une durée d'un an, relancé en 1998 et destiné aux titulaires d'un diplôme universitaire. Au niveau secondaire, où pratiquement toutes les formations se font via des parcours d'un an que l'on intègre après le diplôme, la proportion de stagiaires suivant des parcours en milieu professionnel avait atteint $25 \%$ au milieu des années 2000. Parmi ces programmes de formation, on citera Teach First ( Enseignez d'abord»), association caritative qui a reçu le soutien des plus hauts responsables politiques, toutes tendances confondues, et qui promeut un programme de formation en milieu professionnel à la fois hybride, de petite envergure et s'adressant à une élite. Sur le modèle de Teach for America ( Enseignez pour l'Amérique»), cette association a été conçue pour de jeunes et brillants diplômés prêts à s'engager au minimum deux ans pour enseigner dans des établissements sensibles. Une fois leur qualification en poche, les participants intègrent durant leur deuxième année d'enseignement un programme de formation aux qualités de direction ${ }^{3}$ pour les préparer à embrasser une carrière dans le commerce ou l'industrie ou, s'ils choisissent de rester dans l'enseignement, dans l'encadrement éducatif et la direction d'établissement. L'idéologie politique qui sous-tend l'action menée par Teach First est la quintessence même de la "Troisième voie » prônée par le New Labour. L'association a reçu le soutien du monde des affaires et de l'industrie et possède un large éventail d'ambassadeurs, si bien que les participants au programme sont à même, lorsqu'ils quittent l'enseignement, d'apporter leur contribution au problème de l'éducation en milieu défavorisé par d'autres moyens. Créée à Londres en 2002, puis introduite en régions à partir de 2006, l'association forme aujourd'hui environ $2 \%$ des nouveaux candidats à l'enseignement mais doit développer son activité. Bien qu'elle ne soit pas présente dans les autres nations britanniques, son modèle s'est étendu à de nombreux pays d'Europe et du Commonwealth sous la bannière Teach for All («Enseignez pour tous les publics »).

D’importants changements sont également intervenus récemment dans la structure même de la formation des enseignants du primaire, qui pendant une grande partie de la deuxième moitié du $\mathrm{XX}^{\mathrm{e}}$ siècle relevait du diplôme universitaire d'éducation sanctionnant quatre années d'études (Bachelor of Education

3. « Leadership » dans le texte (NdT). 
Degree). L'introduction de frais de scolarité dans l'enseignement supérieur a rendu les parcours de formation en quatre ans particulièrement onéreux. En outre, il y a eu de nombreuses tentatives de récupération politique faisant valoir que les étudiants intégraient le début de la formation en quatre ans avec un niveau de qualification assez bas, alors que la concurrence très rude pour accéder à la formation des enseignants du primaire après le diplôme universitaire faisait monter le niveau de connaissances. Quelques années plus tard, l'introduction de bourses dans le cadre de la formation post-diplôme a diminué plus encore l'attrait des formations longues. Au milieu des années 2000, la formation longue a été réduite à trois années et ce nouveau format attirait encore $40 \%$ des candidats à la formation, mais dans le primaire le pourcentage total de candidats aux formations en trois et quatre ans est tombé à $35 \%$, alors que les formations post-diplôme d'un an ont atteint $50 \%$ et celles en milieu professionnel, $15 \%$ (McNamara, Brundrett \& Webb, 2008).

\section{LE CADRE RÉGLEMENTAIRE}

Le premier changement en matière de réglementation dans les années 1980 a consisté en un accroissement graduel mais néanmoins important du renforcement de la formation, notamment en termes d'exigences. Dès les années 1990, le nombre de journées obligatoires en établissement pour la formation post-diplôme représentait les deux tiers de la formation sur 36 semaines au niveau secondaire, et la moitié de la formation sur 38 semaines au niveau primaire. Outre le fait que le nombre d'activités hors pratique professionnelle a diminué considérablement, les connaissances et les savoir-faire demandés aux stagiaires se sont faits plus précis et ont vu leur nombre croître avec l'introduction de " compétences" pédagogiques. L'effet conjugué de ces changements a entraîné une pression nettement plus grande sur les volets de formation dispensés par les universités, ainsi qu'une plus grande pression financière globale, en raison de la répartition des financements entre les universités et les établissements scolaires et vu le rôle nettement accru de ces derniers. L'accroissement du renforcement de la formation a entraîné un trop-plein d'effectifs dans les centres et certains aspects jusque là cruciaux de l'élaboration des programmes et du développement professionnel ont été éliminés.

Mais le changement le plus radical et le plus vaste est intervenu lors de la création du Curriculum national pour la formation initiale des enseignants (National Curriculum for Initial Teacher Training) en 1998, suite à une "consultation » qui a suscité "l'inquiétude» de certains universitaires quant à "l'état de la démocratie en Angleterre" (Mahony \& Hextall, 2000, p. 323). Le curriculum a contraint les formations à se conformer à des exigences en termes de durée, d'aménagements pour la formation de partenariats avec les établissements, de sélection des candidats et de processus d'assurance qualité. Dans ce curriculum étaient également définis dans les moindres détails (ce qui aurait été 
inconcevable auparavant) plus de cent critères de réussite que les stagiaires devaient atteindre pour obtenir le grade de professeur certifié (QTS : Qualified Teacher Status). Ces critères concernaient les points suivants : connaissances et compréhension ; préparation des cours, enseignement et gestion de classe ; suivi, évaluation, capacité à consigner et à rendre compte, responsabilité. D'autres exigences professionnelles y figuraient aussi. Il y avait en outre d'autres critères relatifs à la vérification de savoirs disciplinaires définis. En 1999, la formation a été allongée par la mise en place d'une période " probatoire » durant la première année d'enseignement d'un professeur nouvellement certifié. Des critères d'entrée ont été définis afin de formaliser le processus d'évaluation sur trois trimestres, géré par les autorités éducatives locales (Local Authorities) en collaboration avec les établissements employeurs. Les professeurs nouvellement certifiés pouvaient recevoir de l'aide de conseillers tuteurs dans leur établissement d'exercice et bénéficier d'une réduction rémunérée de $10 \%$ de leur temps de présence, afin de prendre part à des activités de développement professionnel.

Les exigences concernant les connaissances disciplinaires ont été accrues davantage encore en 2001, lorsqu'on a introduit des épreuves d'aptitude pour le grade de professeur certifié (QTS skills tests) en mathématiques, anglais et technologies de l'information et de la communication pour tous les stagiaires du primaire et du secondaire. Ces épreuves ont suscité la controverse surtout parce qu'elles étaient centrées sur les savoirs professionnels tels que l'interprétation des données concernant le niveau de connaissances des élèves plutôt que sur les savoirs liés au curriculum. Elles ont également révélé un schisme dans la rivalité des discours sur la "vérification » (auditing) ou "l'examen " (testing) du niveau de connaissances. En 2002, le Curriculum national pour la formation initiale des enseignants a été remplacé par une version qui ne comportait que quarante critères de certification des enseignants (QTS standards). Le nouveau gouvernement du Parti travailliste avait désormais formellement abandonné toute tentative de prescription en matière de pédagogie ou d'inventaire détaillé des savoirs disciplinaires, même si les épreuves d'aptitude au grade de professeur certifié (QTS skills tests) demeuraient en place. La profession a accueilli cette nouvelle mouture de façon plus positive (Simco \& Wilson, 2002), en particulier parce qu'elle mettait nettement l'accent sur les pratiques et les valeurs professionnelles. La troisième mouture du Curriculum, qui date de 2007, a fait l'objet d'un subtil remaniement par rapport à la version de 2002. Les nouvelles réformes du curriculum scolaire se sont retrouvées au premier plan (leur cadre de référence étant connues sous le nom de Every Child Matters, "Chaque enfant compte») et le nombre de critères a été limité à 33 .

L'apprentissage du métier d'enseignant continue toutefois de requérir des connaissances de plus en plus élevées en termes de curriculum, de pédagogie et de compétences. La tâche est encore plus lourde pour les stagiaires du primaire, enseignants "généralistes » qui, en dépit de l'importance durable des matières 
fondamentales (notamment l'anglais et les mathématiques), sont tenus d'être compétents dans onze matières du Curriculum national ${ }^{4}$ (National Curriculum), sachant que les langues vivantes étrangères s'ajouteront bientôt. Le candidat aspirant à être enseignant dans le primaire ou le secondaire est aujourd'hui également tenu d'élargir l'importance centrale donnée aux matières de l'enseignement général pour y inclure les quatre objectifs que se donne le cadre Every Child Matters : contribution à la société, sécurité, santé et bien-être économique. Le futur enseignant doit par ailleurs appréhender un large éventail de contextes professionnels qui inclut le travail avec d'autres intervenants dans la classe ainsi que le travail au sein d'équipes pluridisciplinaires donnant accès aux services de l'enseignement intégré et spécialisé ${ }^{5}$ (integrated and specialist services) - notamment le soutien apporté aux parents et aux familles, la formation des adultes dans les structures de proximité - et, enfin, la promotion de la cohésion sociale au sein de la collectivité.

Le curriculum pour la formation initiale des enseignants de 2007 doit également son importance au fait qu'il s'insérait dans un cadre cohérent de critères professionnels à l'échelle nationale (National Professional Standards) visant à permettre à l'ensemble de la profession enseignante de développer ses compétences, depuis la formation initiale jusqu'à la formation continue destinée aux enseignants les plus compétents et chevronnés. Ce cadre avait été envisagé dix ans plus tôt et était désormais possible car en 2005, dix ans après sa création, l'Agence pour la formation des enseignants a été relancée sous le nouveau nom de l'Agence pour la formation et le développement (Training and Development Agency), dont la mission élargie recouvrait la formation et le développement de l'ensemble de la profession enseignante. La formation initiale des enseignants avait ainsi toutes les chances d'être reléguée au second plan devant l'ampleur de la tâche que l'Agence pour la formation et le développement devait désormais accomplir.

\section{INSPECTION}

Un autre changement de taille pour les universités qui avaient en charge la formation des enseignants avait trait aux mécanismes d'assurance qualité. L’inspection pédagogique, qui s’appuyait auparavant sur le modèle de « l'expertise

4. «L'un des dispositifs important de la réforme de 1988 a été la mise en place d'un curriculum national obligatoire pour les écoles et lycées publics. Il comprend un enseignement de base comportant trois matières fondamentales et six matières principales. À cette formation générale commune s'ajoute un système d'options sur les $25 \%$ du temps restant, afin de permettre aux élèves de préparer leurs options professionnelles. La scolarité se divise en quatre périodes clés à la fin desquelles l'acquisition des connaissances est contrôlée par des tests nationaux » (NdIR). C. de Bouttemont, « Le système éducatif de l’Angleterre et du pays de Galles ", RIE n 33, 2003, p. 139-147.

5. L'enseignement spécialisé s'adresse aux élèves qui rencontrent des difficultés scolaires dans l'enseignement ordinaire ou qui présentent un handicap (physique ou mental, temporaire ou permanent) nécessitant un environnement adapté. Enseignement intégré: les élèves souffrant de troubles de l'apprentissage peuvent dans certains cas assister aux cours de l'enseignement ordinaire (en partie ou entièrement) moyennant une aide apportée par l'enseignement spécialisé (NdT). 
éclairée» (informed connoisseurship), a adopté au milieu des années 1990 le nouveau modèle "techniciste» (Campbell \& Husbands, 2000). Les premières inspections "nouvelle manière " dans le cadre de la formation des enseignants sont apparues à cette époque et ont provoqué chez les enseignants un choc culturel considérable. Le nouveau régime d'inspection annonçait une ère de surveillance et de contrôle qui prétendait proposer une plus grande transparence des critères ainsi qu'une plus grande cohérence dans les appréciations, suscitant de violentes contestations de la part de ceux qui mettaient en doute cette transparence et cette cohérence. Le système de notation (de remarquable à insuffisant) utilisé pour mesurer les critères, sert également de base à l'octroi des dotations aux centres de formation. De ce fait, des notes basses peuvent entraîner des pénalités sous forme de diminution des dotations.

L'enjeu du processus d'inspection constituait un tel poids aux yeux des intéressés que la sous-commission gouvernementale de l'éducation au Parlement, dans une tentative pour contenir les excès des inspections, a recommandé la mise en place immédiate d'un cycle de formation d'au moins quatre ans qui propose un cadre plus léger et différencié. Ce cadre, centré sur la gestion et l'assurance qualité, a été mis en place en 2002, mais il n'est pas apparu aux centres de formation qu'il était sensiblement moins lourd que le précédent (UCET, 2007). De fait, un instrument supplémentaire (une évaluation annuelle) a été introduit en 2008, obligeant tous les centres de formation des enseignants à soumettre aux services de l'inspection un rapport d'auto-évaluation de chaque programme de formation dispensé, identifiant les points forts et les points faibles et mettant l'accent sur la planification des améliorations à apporter. Une analyse relative des coûts et des bénéfices de ce processus reste à effectuer, ainsi qu'une évaluation de son impact global sur l'amélioration de la qualité de la formation. En outre, selon une tendance actuelle au Royaume-Uni qui consiste à utiliser, dans les audits, les données nationales issues des questionnaires étudiants afin d'évaluer les cursus conduisant au diplôme universitaire, l'enquête annuelle réalisée auprès des enseignants nouvellement certifiés est de plus en plus souvent utilisée, en dépit des inquiétudes soulevées par la méthodologie de l'enquête, dans les processus d'inspection et d'évaluation.

En l'an 2000, très peu de formateurs étaient d'avis que le poids combiné de l'inspection et de la notation de la formation dispensée, d'une part, et de l'évaluation du niveau de compétences des stagiaires, d'autre part, avait amélioré la qualité globale de leurs formations (Mahony \& Hextall, 2000). Néanmoins, d'aucuns pourraient avancer que c'est grâce à la rigueur du régime d'inspection et d'évaluation qu'environ $45 \%$ des formations traditionnelles dispensées à l'université étaient qualifiées de remarquables par les inspecteurs en 2007-2008, sur le plan de leur efficacité générale. En comparaison, jusqu'en 2008-2009, le Graduate Teacher Programme, ce programme en pleine expansion de formation en milieu professionnel, a bénéficié d'une tolérance certaine qui lui a permis de 
ne pas être "noté » et de croître malgré des années de rapports d'enquête plus que défavorables. Ainsi, loin de générer des pratiques d'évaluation fiables et cohérentes, l'inspection a émis des messages contradictoires dans sa façon de privilégier tel ou tel parcours de formation. De plus, plutôt que d'évaluer la qualité de l'étendue de toute formation dispensée, l'inspection a été organisée de façon stratégique afin de pousser au changement dans le curriculum et le système scolaire, en mettant l'accent sur un certain nombre d'entreprises liées à l'enseignement et sur des objectifs définis à l'échelon national (McNamara, Brundrett \& Webb, 2008).

\section{PARTENARIAT : L'ESPACE CONTESTÉ}

Certaines universités et certains collèges universitaires avaient, dans les années 1980 et 1990, volontairement accompli des pas de géant pour développer des liens formels avec les établissements scolaires dans le cadre de la formation des enseignants, mais nombreux étaient ceux qui au départ trouvaient simpliste la façon dont le gouvernement décrivait le stagiaire en formation, qui acquiert des compétences pratiques en établissement scolaire et des savoirs disciplinaires à l'université. La prescription de stages de formation obligatoires en établissements scolaires n'était que le début d'un changement de culture bien plus profond, changement exigé des centres de formation par le biais de la mise en place, à la fin des années 1990, de formations en partenariat. Furlong et al. (2000) ont identifié un continuum dans les modèles de partenariat, qui allaient des formations menées par l'université à celles entièrement menées par les établissements. Ils ont avancé l'idée qu'aucun des deux extrêmes ne constitue un véritable partenariat. La mise en place de formations entièrement axées sur l'école et de partenariats en milieu professionnel avait porté gravement atteinte à la notion de partenariat en tant qu'entreprise de collaboration. Dans un cas comme dans l'autre, un partenariat avec l'université n'était pas nécessaire. Un bilan des partenariats, dans lequel l'Angleterre a été comparée à d'autres parties du Royaume-Uni, a montré que « le fait que certains modes d'accès à la profession soient détachés de l'université est peut-être le reflet du niveau relativement bas de l'enseignement dans la culture anglaise "(Brisard et al., 2005). Ce détachement a également entraîné le fait que les qualifications professionnelles et universitaires au métier d'enseignant ont emprunté des voies distinctes. La formation universitaire post-diplôme traditionnelle est sanctionnée soit par le " certificat d'aptitude professionnelle à l'enseignement» (professional certificate of education), au niveau de la licence avec mention en quatre ans (Honours degree), soit par le «certificat post-universitaire d'aptitude professionnelle à l'enseignement " (post-graduate certificate of education), au niveau du master. Ces deux qualifications suivent la recommandation faite par le Conseil général de l'enseignement pour l'Angleterre d'attribuer le grade de Qualified Teacher 
Status ( «statut de professeur certifié »), et malheureusement pour les employeurs, elles portent toutes les deux l'abréviation PGCE. Les parcours de formation en milieu professionnel, en revanche, ne proposaient jusqu'à une période récente que le Qualified Teacher Status, mais certains parcours commencent à proposer des modules de formation qui débouchent sur un diplôme.

En dépit de l'intérêt suscité au plan international, l'Angleterre et le Pays de Galles demeurent toujours les seuls pays où «le partenariat s'est institutionnalisé à l'échelle nationale en tant que principe fondamental de la formation » (Furlong et al., 2006, p. 33), mais compte tenu de la croissance rapide des effectifs de formation, la crise semblait inévitable. Un Projet national de partenariat (National Partnership Project, 2001-2005), doté de larges ressources financières, a été élaboré dans le but d'accroître la capacité du système à placer des stagiaires dans les établissements, à améliorer la qualité de la formation en établissement et à favoriser la collaboration entre les différents partenaires et autres parties intéressées. Lorsque le projet a été abandonné, une fois que le danger imminent semblait s'être dissipé, il n'a guère été évident de déterminer ce que l'on avait appris sur les mécanismes idéologiques, politiques et philosophiques du partenariat, ni de comprendre que le partenariat avait tout simplement été réduit à n'être qu'une tâche technique et rationaliste. L'un des principaux points forts des partenaires universitaires, selon certains, "réside dans la théorisation des fondements épistémologiques et pédagogiques de la formation », si bien qu'en leur absence, "le caractère complexe et contestable des savoirs professionnels n'est plus perçu comme étant au cœur de l'essence même du partenariat; les savoirs professionnels se simplifient... et il s'agit essentiellement de s'intéresser aux pratiques contemporaines à l'école» (Furlong et al., 2006, page 14).

Le 17 juin 2010, Michael Gove, le nouveau ministre de l'Éducation, a annoncé son intention de "réformer la formation des enseignants et de faire passer les enseignants stagiaires des bancs de l'université à la salle de classe » (ministère de l'Éducation)... La profession attend de voir la suite des événements et retient son souffle!

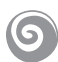

Les tendances centralisatrices et la culture de la responsabilité, qui ont connu une évolution rapide en Angleterre durant les deux décennies des gouvernements conservateurs (couvrant la période des années 1980 et 1990), se sont maintenues, voire intensifiées, à la fin des années 1990 lorsque le Parti travailliste a pris le pouvoir. Certains attribuent le fait que la formation initiale des enseignants est sortie de la relative obscurité dans laquelle elle se trouvait pour revêtir aujourd'hui une importance stratégique, à l'hypothèse émise par les gouvernements successifs que la formation initiale constituait un moyen efficace d'apporter des changements dans le curriculum scolaire et de transformer le 
professionnalisme enseignant (Furlong, 2005). Cela a donné lieu à l'investissement tout à fait opportun de ressources et il y a par ailleurs eu en pratique des innovations de taille, telles que le développement d'un cadre commun d'attentes professionnelles, ainsi qu'une plus grande cohérence dans la qualité des formations dispensées. Néanmoins, il y a eu un certain nombre d'effets indésirables. Premièrement, le manque de contrôle professionnel résultant du degré accru de centralisation a contraint la profession à s'en tenir à une vision à court terme et à subir les lubies du monde politique, et a été source d'instabilité pour les organisations professionnelles et les programmes de formation, rendus ainsi plus vulnérables. Deuxièmement, le niveau de réglementation et de responsabilité ainsi que la mise en rapport des indicateurs de performance avec l'attribution des ressources ont donné lieu à une approche "technique et rationaliste » des résultats et des processus, ont restreint la nature de l'engagement professionnel et créé une "culture du respect de la réglementation » tant chez les stagiaires que chez les formateurs d'enseignants. Troisièmement, les évolutions se sont parfois heurtées à des forces idéologiques contradictoires dans les valeurs et les principes fondamentaux de l'enseignement. Cela pose notamment un problème quand cela a pour conséquence une désarticulation des fondements théoriques et intellectuels des savoirs professionnels et pédagogiques.

\section{Bibliographie}

ALEXANDER R.J. (1984) : 'Innovation and continuity in the initial teacher education curriculum', in R.J. Alexander, M. Craft and J. Lynch (Eds.) Change in Teacher Education: context and provision since Robbins. London: Holt, Rinehart and Winston: 103-160.

BRISARD E., MENTER I. and SMITH I. (2005) : Models of Partnership in Programmes of Initial Teacher Training: a systematic review. Edinburgh: General Teaching Council of Scotland.

CAMPBELL J. and HUSBANDS C. (2000) : 'On the reliability of OFSTED inspection of Initial Teacher Training: a case study', British Journal of Educational Research 26(1): $39-48$.

Department for Education (Ministère de l'Éducation) (2010) : Gove to the National College: 'We have to make opportunity more equal' (Titre de journal : le ministre de l'Éducation Michael Gove s'adresse au Collège National: "Nous devons favoriser l'égalité des chances ") [Disponible sur : http://www.education.gov.uk/news/news/ nationalcollege, consulté le 28 août 2010].

FURLONG J., BARTON L., MILES S., WHITING C. and WHITTY G. (2000) : Teacher Education in Transition: re-forming professionalism? Buckingham: Open University Press.

FURLONG J. (2005) : 'New Labour and teacher education: the end of an era', Oxford Education Review 33(1): 119-134. 
FURLONG J., CAMPBELL A., HOWSON J., LEWIS S. and MCNAMARA O. (2006) : 'Partnership in English teacher education: changing times, changing definitions evidence from the Teacher Training Agency National Partnership Project', Scottish Education Review 37: 32-45.

MCNAMARA O., BRUNDRETT M. and WEBB R. (2008) : Primary Teachers: Initial teacher education, continuing professional development and school leadership development (Primary Review Research Survey 6/3), Cambridge: University of Cambridge Faculty of Education.

MAHONY P. and HEXTALL I. (2000) : Reconstructing Teaching: standards, performance and accountability. London: RoutledgeFalmer.

GTCE (2009): Annual digest of statistics 2008-09. Profiles of registered teachers in England. London: GTCE (Condensé annuel des statistiques 2008-2009 du Conseil général de l'éducation pour l'Angleterre. Profils des enseignants inscrits au registre en Angleterre).

UCET (2007) : Initial Teacher Education Inspection Burdens. London: UCET. (UCET = Conseil des universités pour la formation des enseignants).

SIMCO N. and WILSON T. (2002) : Primary Initial Teacher Training and Education: revised standards, bright future? Exeter: Learning Matters. 